\title{
Expression of the TAF4b gene is induced by MYC through a non-canonical, but not canonical, E-box which contributes to its specific response to MYC
}

\author{
KWESI TEYE ${ }^{1}$, KENGO OKAMOTO ${ }^{1}$, YUJI TANAKA ${ }^{1}$, TOSHIYUKI UMATA ${ }^{2}$, \\ MASAAKI OHNUMA ${ }^{3}$, MASAAKI MOROI ${ }^{3}$, HIROSHI KIMURA $^{4}$ and MAKOTO TSUNEOKA ${ }^{1}$ \\ ${ }^{1}$ Faculty of Pharmacy, Takasaki University of Health and Welfare, Takasaki 370-0033; ${ }^{2}$ Radioisotope \\ Research Center, University of Occupational and Environmental Health, Kitakyushu 807-8555; \\ ${ }^{3}$ Division of Protein Biochemistry, Institute of Life Science, Kurume University, \\ Kurume 839-0861; ${ }^{4}$ Chiba Institute of Science, Choshi 288-0025, Japan
}

Received May 19, 2008; Accepted August 4, 2008

DOI: 10.3892/ijo_00000118

\begin{abstract}
Transcription factor binding sites are short DNA sequences that interact with transcription factors and the proper control of gene expression appears to require the mechanisms including the regulation through the genome context around the transcription factor binding sites. The MYC proteins are central regulators of cell growth. Many genes have been reported to be regulated by MYC through E-box sites. However, the characters of E-box that Myc selects to function are not clear and identification of additional genes controlled by MYC will provide information to completely understand the functions of MYC. Here we report that MYC directly induces TAF4b expression. We mapped the transcription start site and characterized functional promoter elements for MYC response in the TAF4b promoter. There are several E-box sequences near the transcription start site, including canonical (CACGTG) and non-canonical (CGCGTG) ones. We found that c-MYC induces TAF4b expression through one of the non-canonical E-box sites, which is in a highly conserved region of TAF4b promoters in mammals, suggesting the importance of the genome context around the target E-box. When the non-canonical E-box in the TAF4b promoter was mutated to a canonical one, MYC functioned on both Eboxes, while another E-box-binding transcription factor, USF, did so on only the canonical E-box. These results suggest that in addition to the context where the target E-box exists, a sequence within an E-box is involved in the mechanisms by which specific E-box sites are selected by Myc.
\end{abstract}

Correspondence to: Dr Makoto Tsuneoka, Faculty of Pharmacy, Takasaki University of Health and Welfare, Takasaki 370-0033, Japan E-mail: tsuneoka@takasaki-u.ac.jp

Key words: MYC, E-box, promoter, transcription, non-canonical, TAF4b

\section{Introduction}

Transcription factor binding sites are short DNA sequences that interact with transcription factors. Although there are a great number of the candidate binding sites in the genome, the transcription factors function only on limited number of sites. Furthermore, while some transcription factors share their target sequences, the genes that respond to them and their biological functions are different. Thus, the proper control of gene expression requires the mechanisms, by which transcription factors specifically select their target sites. Recent studies suggest that the specificity of protein-DNA interaction depends, at least in part, on the genome context around the target sites. For example, the veracity of a potential target site correlates with the GC content and $\mathrm{CpG}$ island (1), preferential spacing between transcription factor binding sites and transcription start sites (2), co-operation of cis-regulatory molecules (3) and clustering of degenerate transcription factor binding sites near the target sites (4). However, it is still unclear how the target specificity for each transcription factor is achieved.

The $m y c$ family of proto-oncogenes consists of three main genes: $c-m y c, N-m y c$ and $L-m y c$ (5-9). The three genes have different expression patterns but appear to have basically the same biological activity. The accumulating data indicate that the $m y c$ family genes are central regulators of cell growth $(6,7,10-14)$. The MYC protein encoded by myc is a member of the basic helix-loop-helix leucine zipper transcription factors $(6,14)$. MYC functions as an E-box-binding transcription factor and activates transcription of a number of genes (15-24). Although there are a number of E-box sites in the genome, Myc selects only some E-boxes to function on.

Besides proteins in the myc proto-oncogene family, there are other classes of E-box-binding transcription factors, including USF (25) and TFE3 (26). Although these factors also transactivate gene expression through an E-box, their biological functions and the genes that respond to them are different from Myc. However, it is unclear which factors are really responsible for the E-box-binding transcription factors to select the specific target genes. 
Here we report that MYC directly induces the expression of TAF4b. The sequence responsible for this induction is a non-canonical E-box and the surrounding sequence of this element is highly conserved in mammals, confirming the importance of the context where E-box exists. Although this site responded specifically to $\mathrm{MYC}$, it also responded to another E-box binding transcription factor, USF, when the noncanonical E-box was mutated to the canonical one. These results suggest that the sequence within E-box have a role for the selection of the target genes by E-box transcription factors.

\section{Materials and methods}

Cell culture. Human glioblastoma cell line T98G cells and its derivative T98Gmycer-2 cells, which express c-MycER chimeric protein (23), were cultured in Eagle's medium supplemented with non-essential amino acids and $10 \%$ fetal calf serum (FCS). The African green monkey kidney fibroblast line Cos-7 were cultured in Dulbecco's modified Eagle's medium supplemented with $10 \%$ FCS. Human promyelocytic leukemia HL60 cells were cultured in RPMI-1640 medium supplemented with $20 \%$ FCS. The rat colon cancer cell line RCN-9 (Riken Cell Bank, Saitama, Japan) and the human erythroleukemia cell line HEL (Health Science Research Resources Bank, Osaka, Japan) were cultured in RPMI-1640 medium supplemented with $10 \%$ FCS.

Polymerase chain reaction (PCR). PCR amplifications were performed in $50 \mu \mathrm{l}$ EX Taq buffer (Takara, Shiga, Japan) containing 10 pmoles of each primer, 1.2 U EX Taq DNA polymerase and $200 \mu \mathrm{M}$ dNTP.

Rapid amplification of $5^{\prime}$ cDNA ends (5'-RACE) analysis. Reverse transcriptase reaction, double-strand cDNA synthesis and adapter ligation from poly(A) ${ }^{+}$RNA $(1 \mu \mathrm{g})$ of HEL cells were performed using a Marathon cDNA amplification kit (Clontech, Palo Alto, CA) as described previously $(23,24)$. The first PCR was performed using primers, AA287145-1st (5'-TTCTTATGCCTCTTCCTTTCTCCTG-3', a sequence in the 5' terminal region of EST clone AA287145) and the AP1 primer provided by the supplier. The temperature profile was initial denaturing at $94^{\circ} \mathrm{C}$ for $1 \mathrm{~min}$, followed by 25 cycles of denaturing at $96^{\circ} \mathrm{C}$ for $15 \mathrm{sec}$ and annealing and extension at $68^{\circ} \mathrm{C}$ for $3 \mathrm{~min}$. One $\mu \mathrm{l}$ of the first RACE-PCR product diluted 1,000 times was used as the template for nest RACE-PCR. Nest PCR was performed using the AA287145-nest primer (5'-TTGGCAAATGAGGCAGGTAGTAACA-3', which corresponds to a sequence in the upstream region of AA287145-1st of EST clone AA287145) and the AP2 primer provided by the supplier. The temperature profile was initial denaturing at $94^{\circ} \mathrm{C}$ for $1 \mathrm{~min}$, followed by 25 cycles of denaturing at $96^{\circ} \mathrm{C}$ for $15 \mathrm{sec}$ and annealing and extension at $68^{\circ} \mathrm{C}$ for $3 \mathrm{~min}$. The amplified DNA fragment was cloned into a pGEM-T vector (Promega, Madison, WI) (pT/fragment TAF4b) and sequenced. The results of sequencing showed that AA287145 is part of the 3'-UTR of the TAF4b gene.

To obtain information about the 5'-terminal of TAF4b mRNA, 5'-RACE amplification was performed as described above, using the primer TAF4bRACE-1 (5'-GGTCTCGGCT CTTGTTACAGTTTGCTG-3'), a sequence in the 5'-terminal region of TAF4b cDNA reported previously (27) and the AP1 primer provided by the supplier for the first PCR; and the TAF4bRACE-2 primer (5'-GGATTGTCGTGGTGTTGGG GGCTTTCA-3', which corresponds to a sequence in the upstream region of TAF4bRACE-1) and the AP2 primer provided by the supplier for the nest PCR. The amplified DNA fragment was cloned into a pGEM-T vector (Promega) and sequenced.

Reverse transcriptase-polymerase chain reaction ( $R T-P C R)$. Synthesis of single-strand cDNA of HEL cells was performed on total RNA $(1 \mu \mathrm{g})$ using a Superscript first-strand synthesis system (Invitrogen, Carlsbad, CA). One $\mu \mathrm{l}$ (total $20 \mu \mathrm{l}$ ) of the resultant single-strand cDNA was used as the template for PCR. The RT-PCR primers for the amplification were TAF4b coding U (5'-CGGAATTCGAAGCTGCGAGAGGT CGGGCGGGTGTCG-3', a sequence in the 5' untranslated region plus an EcoRI site) and TAF4b coding L (5'-CGGAA TTCGGCAGTAAATAGCAAGGATGTGGATGGA-3', a sequence in the 3' untranslated region plus an EcoRI site). The temperature profile was 35 cycles of denaturing at $98^{\circ} \mathrm{C}$ for $15 \mathrm{sec}$, annealing at $65^{\circ} \mathrm{C}$ for $1 \mathrm{~min}$ and extension at $72^{\circ} \mathrm{C}$ for $2.5 \mathrm{~min}$. The amplified DNA fragment was cloned into a pGEM-T vector (Promega) to produce pT/TAF4b and sequenced. The sequence was confirmed by direct sequencing using the amplified fragment.

Reporter plasmids with a TAF4b genomic DNA fragment. A genomic DNA fragment of the human TAF4b gene, which extends from the promoter region to part of exon 1, was amplified by PCR with 5'-TTTTACCATAACCTCACTTGC TGGAAGGGG-3' and 5'-TGTTGGGGGCTTTCACGGCG ACTATCTG-3'. The 2.2-kb amplified fragment was cleaved with $\mathrm{SacI}$ and $\mathrm{NcoI}$ and inserted into the $4.8 \mathrm{-kb}$ fragment of pGL3 containing firefly luciferase (Promega) cut with SacI and $N c o$ I to produce pTAF4b(W)luci. The $0.6 \mathrm{kp}$ fragment of TAF4b(W)luci cut with $S m a I$ and $N c o$ I was inserted into the 4.8-kb fragment of pGL3 cut with SmaI and NcoI to produce pTAF4b(dSma)luci. The $0.6 \mathrm{kp}$ fragment of TAF4b(W)luci cut with $X h o I$ and $N c o I$ was inserted into the $4.8-\mathrm{kb}$ fragment of pGL3 cut with $\mathrm{XhoI}$ and $\mathrm{NcoI}$ to produce pTAF4b(dXho) luci. pTAF4b(dSma)luci was cleaved with XhoI and NcoI, blunted with Klenow enzyme and self-ligated to produce pTAF4b(Sma-Xho)luci. pTAF4b(dSma)luci was also cleaved with $P v u I$ I and $N c o$ I, blunted with Klenow enzyme and selfligated to produce pTAF4b(Sma-Pvu)luci.

Further deletion mutants, pTAF4b(117Pvu)luci, pTAF4b (114Pvu)luci, pTAF4b(102Pvu)luci, pTAF4b(73Pvu)luci, pTAF4b(43Pvu)luci, pTAF4b(Sma118)luci, pTAF4b (Sma87)luci, pTAF4b(Sma74)luci, pTAF4b(Sma68)luci, pTAF4b(Sma59)luci and pTAF4b(Sma45)luci were produced by PCR with appropriate primers using pTAF4b(Sma-Pvu) luci as a template and the subsequent introduction of the resultant fragments into a pGL3 vector.

Mutation was introduced at E-box sites of pTAF4b(W)luci using a Gene Editor ${ }^{\mathrm{TM}}$ in vitro site-directed mutagenesis system (Promega) to produce pTAF4b(mE1/2)luci, in which two E-boxes (CACGTG elements) were mutated to CACCTG. Mutation was also introduced at non-canonical E (NCE)-box sites (CGCGTG) of pTAF4b(Sma-Pvu)luci to produce 
pTAF4b(Sma-Pvu mNCE1/2)luci, pTAF4b(Sma-Pvu mNCE1)luci, and pTAF4b(Sma-Pvu mNCE2)luci, in which one or two NCE-boxes (CGCGTG elements) were mutated to CGCCTG. The non-canonical E (NCE)-box site 2 (NCEbox 2) in pTAF4b(Sma-Pvu)luci was mutated to CACGTG to produce pTAF4b(Sma-Pvu CanoE2)luci. The DNA sequences in the produced plasmids were confirmed by sequencing.

Sequence of the rat and monkey TAF4b promoters. A $1.2 \mathrm{~kb}$ fragment of the rat TAF4b promoter was isolated as follows: First, the mouse DNA sequence homologous to the human TAF4b gene was selected (GenBank Accession no. AK012135). The two regions in the mouse DNA sequence whose nucleotide sequences exactly match the human TAF4b genomic sequences were selected, and oligonucleotides rat TAFII-F (5'-GCGCACGTGTGAGCGCCGCTGAGG-3') and rat TAFIIPF-1 (5'-CTGACAGGAGCCTTAGTCAC-3') were synthesized. With these oligonucleotides as primers, the rat TAF4b genomic DNA was amplified using rat colon cancer cell RCN-9 genomic DNA as a template. The amplified DNA fragment was sequenced and the upstream region of this sequence was further cloned by promoter find kit (Clontech) with $\operatorname{DraI}$ and $S s p \mathrm{I}$ libraries as templates using primers rat TAFIIPF3 (5'-GCCGGGGCGGGCGGCAGGAGACTCG-3') and rat TAFIIPF4 (5'-CGGGCACTCCCTCAGCGGCGCT CAC-3'), whose sequences were in the rat TAF4b DNA sequence identified above and AP1 and AP2. The amplified rat $\mathrm{TAF} 4 \mathrm{~b}$ genomic fragments were sequenced.

The monkey TAF4b promoter was amplified with TAFprimer 1 (5'-GACACAAGGAGAGGAACACGGATGC-3') and TAF-primer 2 (5'-GCCTGGGCTGCCCCCGGAGCGA CAC-3') matching the human TAF4b gene using the genomic DNA of Cos-7 cells as a template and sequenced.

Other plasmids. pRL-CMV containing the Renilla reniformis luciferase gene under control of the CMV promoter was purchased from Promega. CMV-c-Myc, pCMV-USF and pCMV-TFE-3 were gracious gifts from Dr Martin Eilers (28).

$R N A$ preparation. Total RNA was isolated from cells by the acid guanidium thiocyanate-phenol/chloroform extraction method using a DEPC-treated RNA preparation solution set (Nakalai, Kyoto, Japan).

Differential display using DNA chip (cDNA micro array). cDNA micro array was performed as described previously (23). Total RNA was isolated from untreated T98Gmycer-2 cells and from cells treated by 4-hydroxytamoxifen (OHT) for $20 \mathrm{~h}$. Poly $\left(\mathrm{A}^{+}\right) \mathrm{RNA}$ was recovered and subjected to differential display using a DNA chip (Incyte Genomics, Palo Alto, CA). About 9,000 kinds of cDNAs including expressed sequence tag (EST) clones were plated on the chip (UniGEM Human V Ver. 2).

Northern blot analysis and preparation of probe DNAs. RNA was electrophoresed in an agarose gel containing formaldehyde, transferred to Hybond-N (Amersham Biosciences) and probed with ${ }^{32} \mathrm{P}-$ labeled cDNA. Probes were labeled with $\left[\alpha^{-32} \mathrm{P}\right]-\mathrm{dCTP}$ using a Multiprime labeling kit (Amersham Biosciences). The results were quantified using a BAS2000 image analyzer (Fuji Photo Film, Tokyo, Japan). The DNA probe for c-myc was described previously (18). The DNA probe for TAF4b was a $0.6 \mathrm{~kb} S a c \mathrm{I}$ fragment from $\mathrm{pT} /$ fragmentTAF4b.

Transient expression assay. Transient expression reporter assay in T98Gmycer-2 cells was performed as described previously (23). The effects of MYC and other transcription factors on the TAF4b promoter were investigated by transfection of the plasmids encoding the transcription factors. T98G cells were grown in medium supplemented with $10 \%$ FCS. Cells $\left(3 \times 10^{4}\right)$ were plated into a dish (12-well plate; well diameter $22 \mathrm{~mm}$ ) and cultured for 20-24 h. Transfections were carried out using FuGENE 6 reagent (Roche Diagnostics, Indianapolis, IN) with $1 \mu \mathrm{g}$ of the reporter plasmids, $20 \mathrm{ng}$ of pRL-CMV as an internal transfection marker and various amounts of plasmids expressing the transcription factors. The total amount of DNA in each transfection was kept constant by adding the empty expression vector. One day later, cells were collected and the luciferase activities were determined as described previously (18).

Chromatin immunoprecipitation. Chromatin immunoprecipitation assay was performed basically as described previously (23). Immunoprecipitated DNA fragments were detected by PCR. The PCR primers were 5'-GACACAAGG AGAGGAACACGGATGC-3' (TAF4b-primer 1) and 5'-GCC TGGGCTGCCCCCGGAGCGACAC-3' (TAF4b-primer 2), which amplify a 521-bp fragment containing the transcription start site of the human TAF4b gene (TAF4b primers) and 5'-TTACAGGTAAGCCCTCCAATGACC-3' and 5'-GCAA AGCTACCATTTAGGAACCC-3', which amplify the genomic sequence of a region containing an E-box in chromosome 22 (control primers). This E-box is located in a chromosomal region without any detectable genes (20).

Antibodies. Antibody-1 against c-MYC was described previously (18). Anti-c-MYC antibody-2 (N262) (Santa Cruz Biotechnology, Santa Cruz, CA) was purchased.

The sequence information. The sequence data in this study have been submitted to the DDBJ databases under accession No. AB234096 for the DNA sequence of full-length human TAF4b, accession No. AB234097 for the DNA sequence of the human TAF4b promoter, accession No. AB234098 for the DNA sequence of the rat TAF4b promoter and accession No. AB234099 for the DNA sequence of the monkey TAF4b promoter (Cos-7 cells).

\section{Results}

$c-M Y C$ induces the expression of TAF4b. To conditionally induce c-MYC activity, the estrogen-inducible MYC system $(23,29)$ was used. The chimeric protein c-MycER consists of human c-MYC and the estrogen-binding domain of the human estrogen receptor. c-MycER anchors to cytoskeletal components of cells in the absence of estrogen. When estrogen or its analogous molecule 4-hydroxytamoxifen (OHT) binds to the chimeric protein, it becomes free to function as c-MYC. The human glioblastoma cell line T98G was used as the 


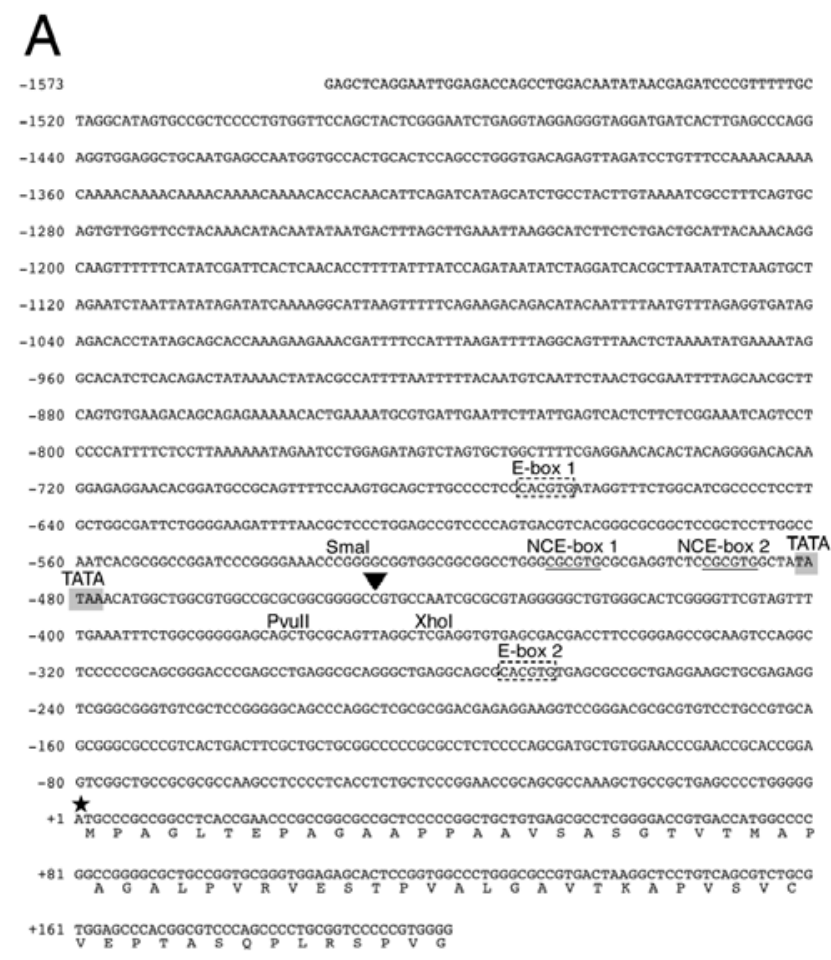

\section{B}

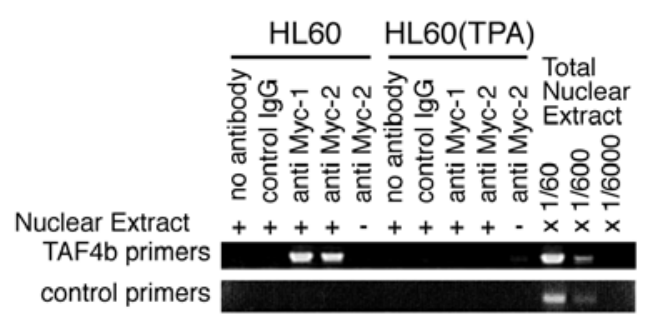

Figure 1. The genomic DNA sequence of the human TAF4b promoter and chromatin immunoprecipitation experiments. (A) The genomic DNA sequence of the human TAF4b promoter and part of exon 1 . The amino acid sequence of the N-terminal end, which had not been reported, is also shown under the DNA sequence with one letter notation. The transcription and translation start sites are indicated by an arrowhead and an asterisk, respectively. E-box sites, E-box 1 and E-box 2 (surrounded by dotted lines), non-canonical E (NCE)-box sites, NCE-box 1 and NCE-box 2 (underlined), TATA box sequence (shaded), a SmaI site (CCCGGG), a PvuII site (CAGCTG), and a XhoI site (CTCGAG) are also shown. (B) Chromatin immunoprecipitation using anti c-MYC antibody. HL60 cells in the proliferating phase (TPA) and those treated with $10 \mathrm{nM}$ TPA for $24 \mathrm{~h}$ (+TPA) were fixed with $1 \%$ formaldehyde, nuclear extracts were collected, and chromatins were immunoprecipitated using antibodies against c-MYC (anti-c-MYC antibody-1 reacting with the N-terminal half of c-MYC and anti-c-MYC antibody-2 reacting with the C-terminal half of c-MYC), control antibody, or no antibody (no antibody). In the presence of TPA, c-myc expression was greatly reduced (Fig. 2A). Mock immunoprecipitation, in which no nuclear extract was added, was also performed (nuclear extract -). Following DNA purification, samples were subjected to PCR with primers designed to amplify a DNA fragment of the TAF4b promoter region (TAF4b primers) or primers to specifically amplify a DNA fragment containing a chromosome 22 E-box (control primers) (20).

parental cell into which ectopic c-MYC activity was introduced. A T98G cell line expressing c-MycER protein (T98Gmycer-2 cells) was established previously (23). Total RNAs from T98Gmycer- 2 cells in an exponentially growing phase in the presence or absence of OHT for $20 \mathrm{~h}$ were subjected to cDNA micro array analysis. Specific signals for
MYC target genes, ornithine decarboxylase (17), nucleolin (19) and mina53 (23) were increased by 2.6-, 1.6- and 1.9-fold, respectively, with c-MycER activation. These results suggest that MYC target genes could be detected in this experimental system.

We found that the signal for EST clone AA287145 was stimulated by 2.3-fold with c-MYC activation. cDNA encoding the 5' upstream part of EST clone AA287145 was isolated using the 5'-RACE protocol from a library of human erythroid leukemia (HEL) cells, and it was found that AA287145 was part of the 3' non-coding region of the TAF4b gene (previously called $\left.\mathrm{TAF}_{\mathrm{II}} 105\right)$ (27). Because the translation and transcription start sites of the human TAF4b gene had not been reported, we isolated cDNA encoding the 5'-end of the transcript by 5'-RACE experiments. The transcription start site was mapped on the TAF4b genomic sequence, which is indicated by an arrowhead in Fig. 1A. Using primers consistent with the $5^{\prime}$ and 3' sequences of TAF4b mRNA, a 3.2-kb length cDNA was amplified from total RNA of HEL cells by RT-PCR protocol and sequenced. Although the entire nucleotide sequence was almost the same as the previously reported one (27), we found an additional 66-amino acid sequence extending from the $\mathrm{N}$-terminal amino acid glycine of TAF4b reported previously (Fig. 1A), and several amino acids were different (the sequence was deposited in GenBank as accession no. AB234096). The cDNA clone encodes a protein of 862 amino acids with a predicted molecular weight of $91090.52 \mathrm{Da}$ and PI of 9.59 .

Expression of TAF4b $m R N A$. Human promyelocytic leukemia HL60 cells are terminally differentiated by 12-O-tetradecanoylphorbol-13-acetate (TPA), in which the c-myc expression level decreases $(30,31)$, after a transient stimulation (32). This experimental system was used to investigate whether MYC-target genes are affected during the shut-off of MYC $(33,34)$. As shown in Fig. 2A, the level of c-myc mRNA had doubled at $1 \mathrm{~h}$ after addition of TPA, then started to decline at $3 \mathrm{~h}$, and reached one-fifth at $7 \mathrm{~h}$. The level of TAF4b mRNA had increased to one and a half-fold at $3 \mathrm{~h}$ after addition of TPA. At $6 \mathrm{~h}$ it started to decline, and reached onetenth at $9 \mathrm{~h}$. These results show that the expression of TAF4b closely followed that of c-myc.

Next, the effect of c-MYC activation on TAF4b mRNA was investigated (Fig. 2B) using the MycER system. The level of TAF4b mRNA had increased to 2- and 3-fold at 3 and $7 \mathrm{~h}$, respectively, in OHT-treated T98Gmycer- 2 cells. The OHT treatment of $\mathrm{T} 98 \mathrm{G}$ parent cells did not stimulate the TAF4b mRNA level. As shown in Fig. 2C, induction of TAF4b mRNA by OHT in T98Gmycer- 2 cells was maintained even in the presence of the protein synthesis inhibitor cycloheximide. Treatment with cycloheximide had little effect on the TAF4b mRNA level in T98G parent cells (data not shown). These results indicate that the TAF4b gene is a direct target of MYC.

c-MYC binds to the TAF4b promoter region in vivo. To examine c-MYC protein binding to the endogenous TAF4b gene in vivo during proliferation of HL60 cells, chromatin immnoprecipitation was performed as described previously $(35,36)$. After immunoprecipitation, enrichment of the 
A
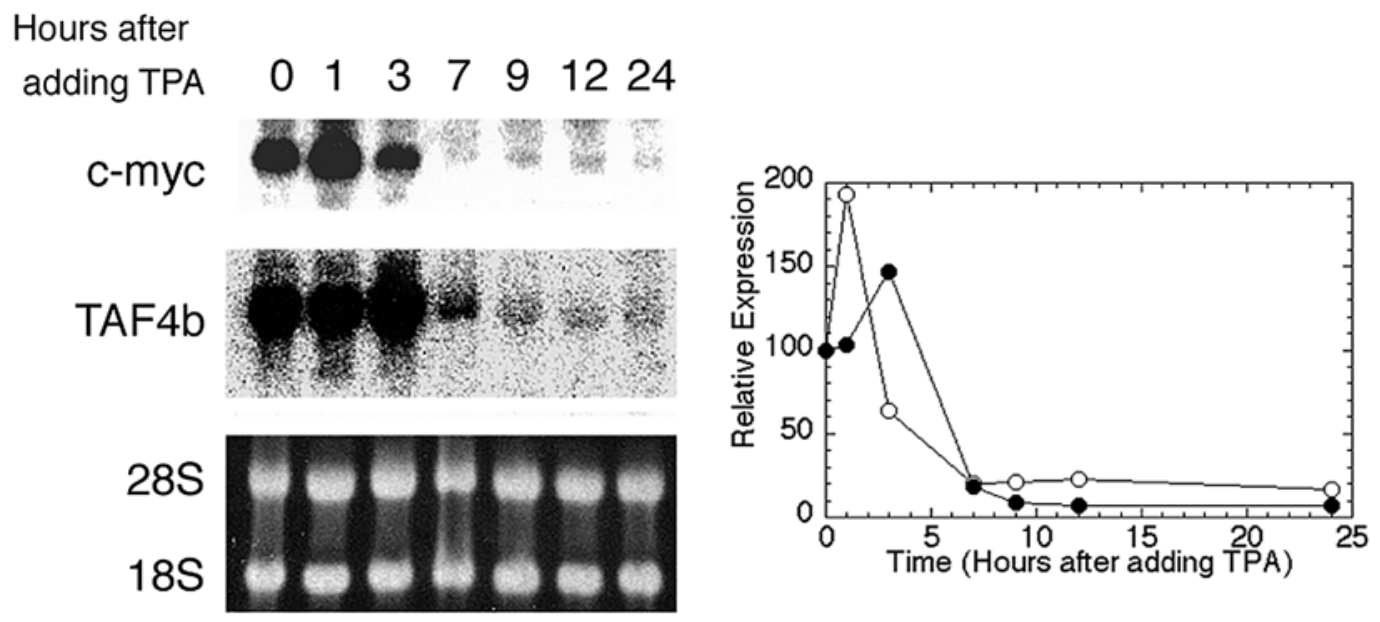

B

\section{T98G}
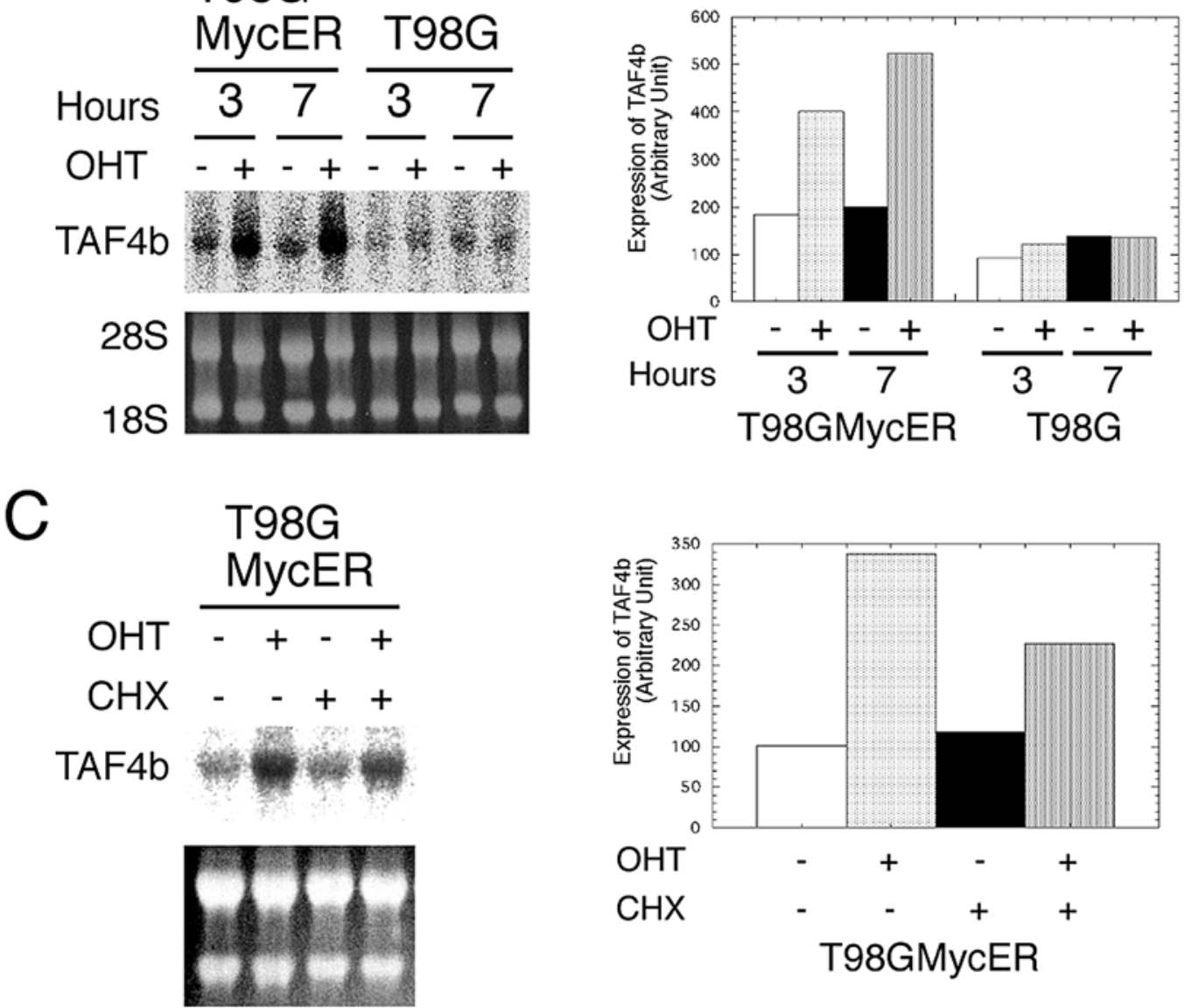

Figure 2. Expression of TAF4b mRNA. (A) The changes of TAF4b and c-myc mRNA in human promyelocytic leukemia HL60 cells after TPA treatment. RNA was isolated at the indicated time points after exposure to $10 \mathrm{nM}$ TPA and analyzed by Northern blotting to detect TAF4b and c- $m y c$ mRNAs (left). $28 \mathrm{~S}$ and $18 \mathrm{~S}$ ribosomal RNAs are also shown. The results were quantified and plotted (right). (B) Increase of TAF4b mRNA levels in T98G cells by activation of MycER protein. After T98Gmycer-2 cells (T98GMycER) and parental cells (T98G) were cultured in the medium supplemented with $0.25 \%$ serum for 40 h, cells were cultured in the presence $(+)$ or absence $(-)$ of $200 \mathrm{nM} \mathrm{OHT}$. RNA was isolated at the indicated time points and analyzed by Northern blotting to detect TAF4b mRNA (left). $28 \mathrm{~S}$ and $18 \mathrm{~S}$ ribosomal RNAs are also shown. The results were quantified and expressed as a bar graph (right). (C) The increase of TAF4b mRNA levels due to activation of MycER protein was maintained in the presence of the protein synthesis inhibitor. After T98Gmycer-2 cells were cultured in the medium supplemented with $0.25 \%$ serum for 40 h, cells were treated with 200 nM OHT for 8 h. RNA was isolated and analyzed by Northern blotting to detect TAF4b mRNA (left). When indicated as + in the row of CHX, $20 \mu \mathrm{g} / \mathrm{ml}$ cycloheximide was added 20 min before the addition of OHT. $28 \mathrm{~S}$ and $18 \mathrm{~S}$ ribosomal RNAs are also shown. The results were quantified and expressed as a bar graph (right). 
A
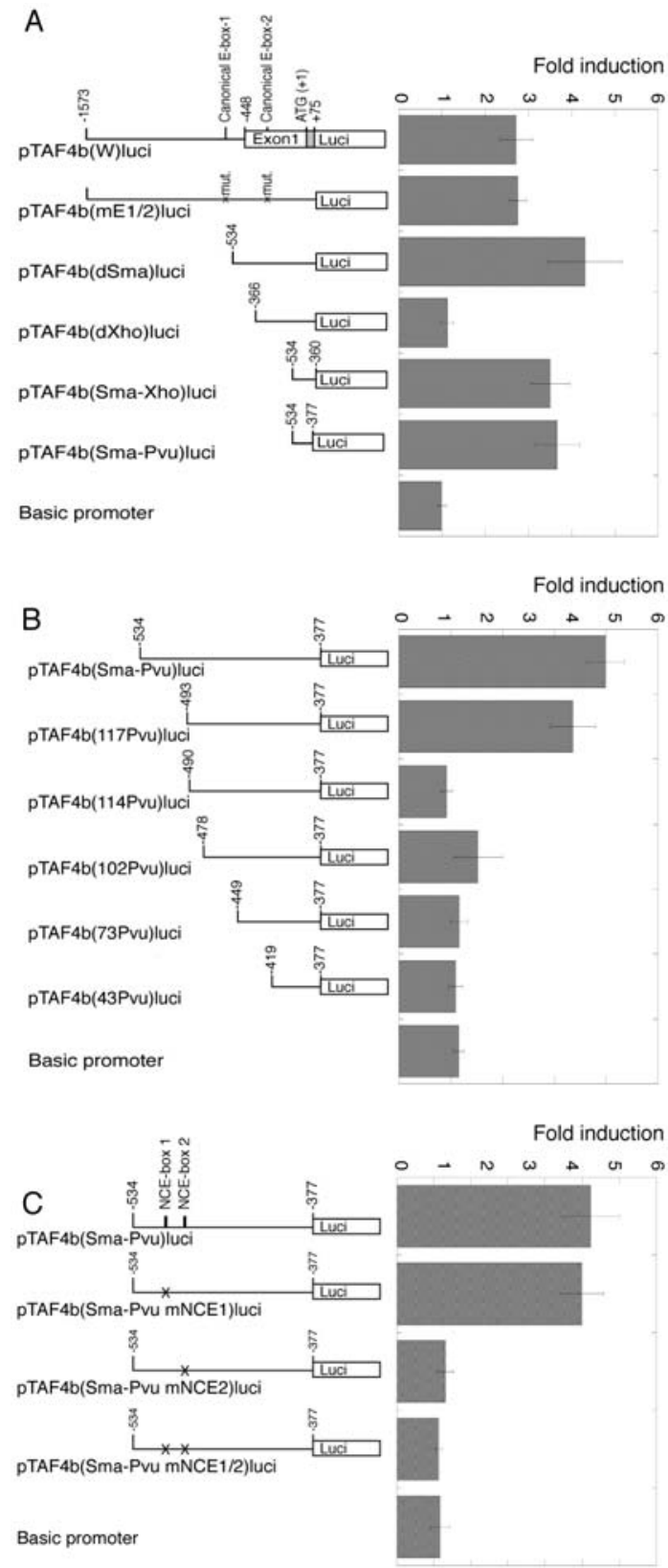

Figure 3. Transient expression from the TAF4b promoter. (A) The reporter plasmid $[\mathrm{pTAF} 4 \mathrm{~b}(\mathrm{~W})$ luci] was constructed to detect the TAF4b promoter activity. In pTAF4b(mE1/2)luci, two E-boxes (CACGTG elements) of pTAF4b(W)luci were mutated to CACCTG (see Fig. 1A). Deletion mutants of pTAF4b(W)luci, pTAF4b(dSma)luci, pTAF4b(dXho)luci, pTAF4b(SmaXho)luci, and pTAF4b(Sma-Pvu)luci were also produced. T98Gmycer-2 cells were transfected with the reporter plasmids. One day later, OHT was added at the final concentration of $200 \mathrm{nM}$ to activate the MycER chimeric protein, and cells were further cultured for $17 \mathrm{~h}$. Cell extracts were analyzed for activities of firefly luciferase. The promoter activities were determined as the ratio to activities without MycER activation. To normalize the effect of OHT not through MycER activation, the values from T98Gmycer-2 cells were divided by those from T98G parent cells, which were treated by exactly the same procedures as T98Gmycer- 2 cells described above. The basic promoter was a pGL3 plasmid without any insert. Values are the means of four separate experiments. The bars indicate the standard errors. (B) $\operatorname{pTAF} 4 \mathrm{~b}(\mathrm{Sma}-\mathrm{Pvu})$ luci were deleted from the 5 '-end to produce pTAF4b(117Pvu)luci, pTAF4b(114Pvu)luci, pTAF4b(102Pvu)luci, pTAF4b(73Pvu)luci, and pTAF4b(43Pvu)luci. The promoter activation by MYC was measured as described in ' $\mathrm{A}$ '. (C) Non-canonical E-box sites, NCE-box 1 and NCE-box 2 (see Fig. 1A) of pTAF4b(Sma-Pvu)luci were mutated to produce pTAF4b(Sma-Pvu mNCE1)luci, pTAF4b(Sma-Pvu mNCE2)luci, and pTAF4b(Sma-Pvu mNCE1/2)luci, in which one or both non-canonical E-boxes (CGCGTG elements) were mutated to CGCCTG. The promoter activation by MYC was measured as described in ' $\mathrm{A}$ '. endogenous TAF4b gene fragment in each sample was monitored by PCR using primers that specifically amplify DNA around the transcription initiation site in the TAF4b gene. As shown in Fig. 1B, two different anti-c-MYC antibodies immunoprecipitated the TAF4b DNA fragment from HL60 cells in the proliferating phase, while the same antibodies did not immunoprecipitate detectable levels of DNA fragments from cells treated with TPA, in which c-myc expression became very low. Enrichment of TAF4b genomic DNA fragments is dependent on c-MYC binding to the TAF4b gene because a nonspecific antibody did not immunoprecipitate TAF4b DNA fragments. Additionally, binding of c-MYC detected in the TAF4b gene is specific, since antibodies against c-MYC did not enrich the genomic DNA fragment containing an E-box that is located in a chromosomal region without any detectable gene in chromosome 22. These results demonstrate that the TAF4b gene around the transcription initiation site is bound by c-MYC specifically at the proliferating phase of HL60 cells.

Identification of c-MYC-responsive element in the TAF4b promoter. To determine the DNA element(s) through which c-MYC controls the expression of TAF4b, the promoter region of the human TAF4b gene was isolated. In Fig. 1A, 'A' in the first ATG was designated as +1 (indicated by an asterisk). The transcription initiation site was at $-460 \mathrm{bp}$, which is indicated by an arrowhead in Fig. 1A. The TATA box sequence was found $\sim 30$ bp upstream of the transcription initiation site.

To investigate the activity of the TAF4b promoter, the genomic DNA fragment including a 1.6-kp region from the upstream region of exon 1 to part of exon 1 was ligated to firefly luciferase cDNA to construct a reporter plasmid, pTAF4b(W)luci (Fig. 3A). Transient expression assays in T98Gmycer-2 cells indicated that the DNA fragment has promoter activity. After c-MycER activation by OHT, the luciferase activity was increased up to 2.5 -fold (Fig. 3A). This stimulation is comparable to that observed for an increase in the TAF4b mRNA level by OHT in T98Gmycer-2 cells (Fig. 2B).

There are two canonical E-box sites (CACGTG elements) near the transcription initiation site. We designated the first and second CACGTG elements as E-box 1 and E-box 2, respectively. Since it had been reported that CACGTG elements function as MYC-responsive elements in many MYC-responsive genes, we examined whether the E-box sites were involved in activation of the promoter activity by c-MYC. When E-boxes 1 and 2 were mutated, however, luciferase activity was still increased by MYC as in the wild-type reporter plasmid (Fig. 3A, lane for pTAF4b(mE1/2)luci).

Next, the region responsible for MYC stimulation was investigated by using deletion mutants (Fig. 3A). While deletion of the region between -1575 and -535 [pTAF4b (dSma)luci ] did not decrease stimulation of the promoter by MYC, deletion of the DNA fragment between -1575 and -367 [pTAF4b(dXho)luci] abolished MYC stimulation, suggesting the importance of the region between -534 and -367 . Then we constructed two additional reporters, which contained the sequences between -534 and -360 [pTAF4b(Sma-Xho)luci] and -534 and -377 [pTAF4b(Sma-Pvu)luci], respectively. The expression of these two reporters was well stimulated by MYC. 
A

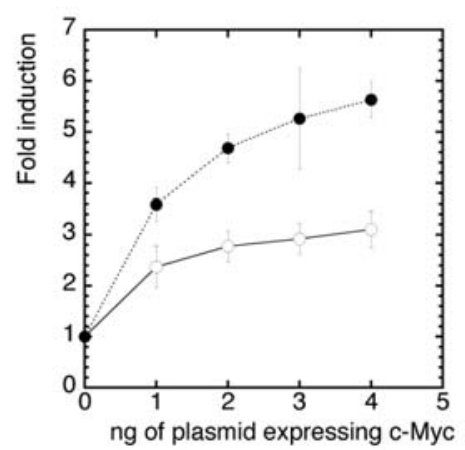

B

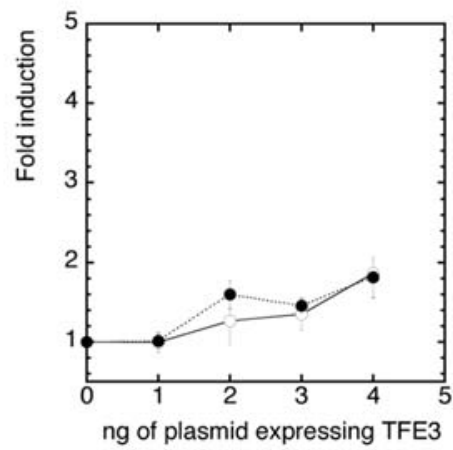

C

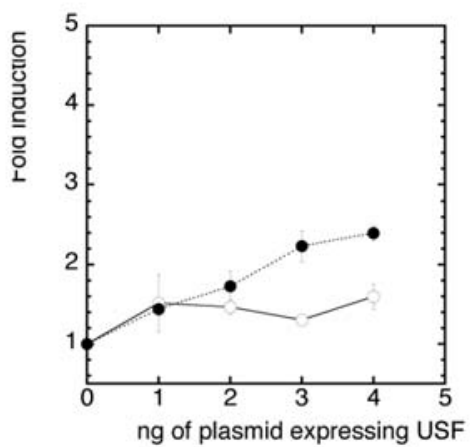

Figure 4. Comparison of the wild-type TAF4b promoter to the one with the canonical E-box. (A-C) The effects of c-MYC, TFE3, and USF expression on the TAF4b promoter activity, pTAF4b(Sma-Pvu)luci ( $)$ and pTAF4b(Sma-Pvu CACGTG)luci (•), in which NCE-box 2 (CGCGTG element) was mutated to the canonical one (CACGTG), were examined. T98G cells were transfected with the reporter plasmids and pCMV-c-Myc (A), pCMV-TFE3 (B), or pCMV-USF (C), together with pRL-CMV (for standardization of the transfection efficiency). Luciferase activities were expressed as the ratio to expression without the effector plasmids. The amounts of the effector plasmids are shown on the X-axis. Values are the means of four separate experiments. The bars indicate the standard errors.

To find the responsible element for MYC-activation, the DNA fragment in the reporter [pTAF4b(Sma-Pvu)luci] was deleted sequentially from the 5'-end of the TAF4b promoter (Fig.3B). While the deletion of the region between -534 and -492 [pTAF4b(117Pvu)luci] marginally reduced the elevation by MYC, deletion of -534 to -490 [pTAF4b(114Pvu)luci] abolished the elevation by MYC. The expression by the reporters with further deletions [pTAF4b(102Pvu)luci, pTAF4b(73Pvu)luci and pTAF4b(43Pvu)luci] was not elevated by MYC. These results suggest the importance of the region between -534 and -490 , especially between -492 and -490 .

The region between -492 and -490 is in a non-canonical E-box element CGCGTG (-492 to -487 in Fig. 1A). It has been reported that c-MYC could bind this sequence (6). Then we tested whether CGCGTG elements were involved in activation by MYC. There are two CGCGTG elements very near the TATA box sequence (Fig. 1A). We designated the first and second CGCGTG elements as non-canonical E (NCE)-box 1 (-509 to -504$)$ and NCE-box 2 (-492 to -487$)$, respectively. We constructed the reporter plasmids pTAF4b(Sma-Pvu, mNCE1)luci, pTAF4b(Sma-Pvu, mNCE2)luci and pTAF4b (Sma-Pvu, mNCE1/2)luci, whose one or both CGCGTGs were mutated to CGCCTG (Fig. 3C). While mutation of NCE-box 1 had little effect on the stimulation of promoter activity by c-MYC, mutation of NCE-box 2 or of both NCE-box 1 and NCE-box 2 completely abolished the MYC-stimulation. These results indicate that c-MYC transactivates the TAF4b promoter through NCE-box 2.

The response of CGCGTG and CACGTG elements to E-box binding transcription factors. In many genes, a sequence of MYC responsive E-box is CACGTG, rather than CGCGTG. To test whether the sequence CGCGTG has any biological significance, NCE-box 2 (CGCGTG) was mutated to the canonical E-box (CACGTG). The promoter with CACGTG element was stimulated by c-MYC and the elevation was higher than that of the wild-type promoter (Fig. 4A).

Besides proteins in the $m y c$ proto-oncogene family, there are other E-box-binding transcription factors, including USF
(25) and TFE3 (26). Then we examined how these E-boxbinding transcription factors responded to CACGTG and CGCGTG elements. As shown in Fig. 4B and C, the wild-type TAF4b promoter was hardly stimulated by TFE3 and USF. These results suggest that the TAF4b promoter specifically responds to MYC. However, when the CGCGTG element was mutated to the canonical CACGTG, the expression from the TAF4b promoter was stimulated by USF. TFE3 still hardly stimulated the expression from the reporter.

The MYC-responsive E-box element and the surrounding sequence in the TAF4b promoter is conserved in mammals. Since the sequence between -493 and -461 appears to be critically important for MYC activation, it was curious to test whether this sequence also exists in other species. We isolated a $1.2 \mathrm{~kb}$ fragment of the rat TAF4b genomic DNA containing the promoter region, and sequenced it. Fig. 5A shows the difference in the nucleotide sequences between the human and rat TAF4b promoter regions. The nucleotide diversity was calculated using the average number of nucleotide differences in sliding and overlapping windows of $100 \mathrm{bp}$, placed at $10 \mathrm{bp}$ intervals. In this analysis, an insertion/deletion (indel) was treated as a single mutation. The minimum values of nucleotide diversity, namely the strongest homology, were observed around NCE-box 2 (location 792). Low values of nucleotide diversity were also observed around location 1000. This may be attributed to the fact that, in those locations, there are several large indels, which caused the nucleotide diversity to be underestimated. When the human TAF4b promoter sequence was compared to that of mouse, whose DNA sequence was recently deposited in the GenBank (accession no. AC133172.4), similar results were obtained (data not shown).

We isolated the TAF4b promoter DNA fragment of monkey from African green monkey kidney fibroblast Cos-7 cells and sequenced it. The nucleotide sequences around NCE-box 2 in human, monkey, rat and mouse are compared in Fig. 5B. The 29 bp sequence containing NCE-box 2 and the TATA box element are completely conserved among the four species studied. 


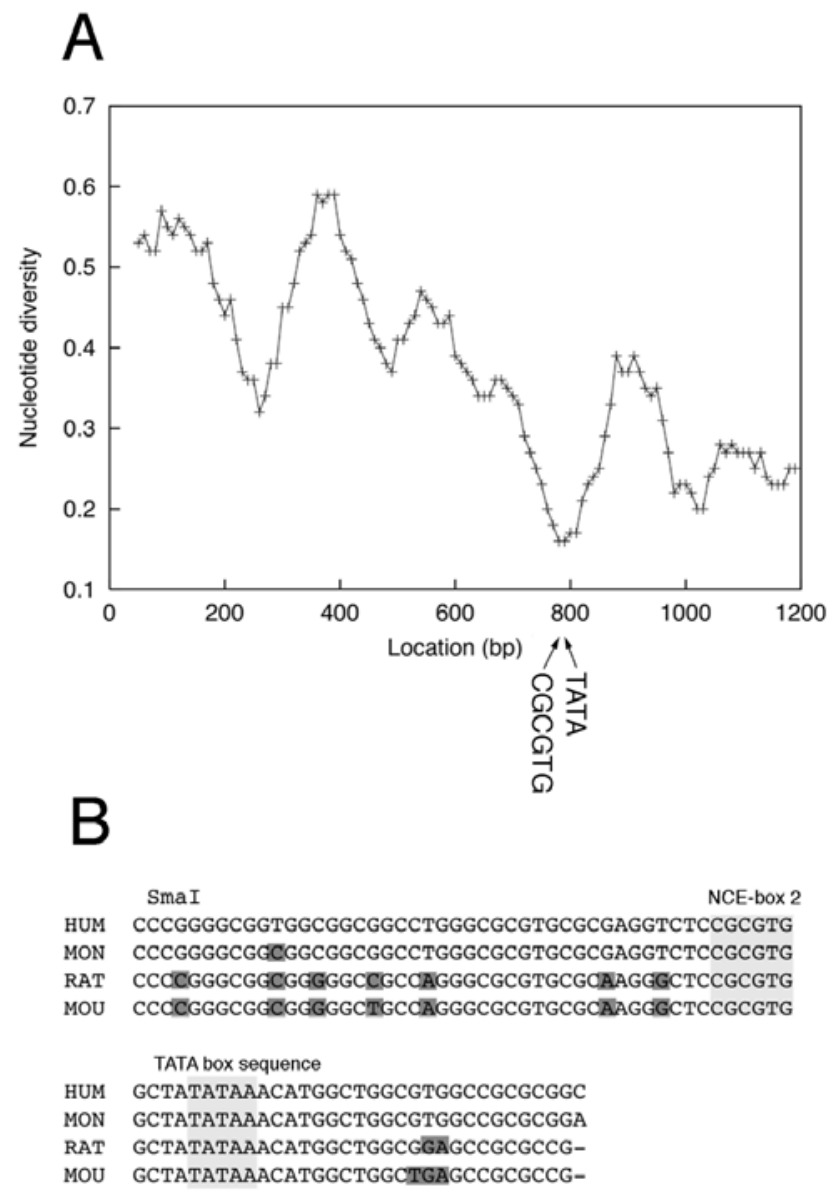

Figure 5. TAF4b promoters of other species. (A) Comparison of the human and rat TAF4b promoter sequences. In this analysis, highly homologous regions give a low score (see text). The position of NCE-box 2 (CGCGTG) and the TATA box sequence (TATA) are indicated (see Fig. 1A). (B) Comparison of the sequences around the CGCGTG element in the human (HUM), monkey (MON), rat (RAT), and mouse (MOU) TAF4b promoters. NCE-box 2 and TATA box sequences are lightly shaded. The nucleotides that are different from the human TAF4b promoter are darkly shaded. The SmaI site in the human promoter is also shown.

\section{Discussion}

c-MYC induces TAF4b expression through an E-box site, which is in a highly conserved region of TAF4b promoters in mammals. We found evidence that MYC directly induces the expression of TAF4b. After treatment with TPA, the expression of TAF4b mRNA closely followed that of c-myc in HL60 cells (Fig. 2A). Stimulation of the expression of TAF4b mRNA was observed when c-MYC was activated in the c-MycER chimeric protein (Fig. 2B). This induction was maintained even in the presence of a protein synthesis inhibitor (Fig. 2C), indicating that c-MYC directly controls the expression of TAF4b. c-MYC protein bound the TAF4b promoter in vivo in proliferating HL60 cells (Fig. 1B). The TAF4b promoter activity was elevated by c-MYC (Fig. 3). This elevation was mediated through an E-box (NCE-box 2; a CGCGTG element) (Fig. 3).

All E-boxes are not always MYC-responsive elements. The MYC-responsive E-box in the TAF4b promoter exists in the most conserved region between human and rat (Fig. 5A) where GC content is high (Fig. 1A). The 29 bp-nucleotide sequence covering the MYC-responsive element and TATA box is completely conserved in human, monkey, rat, and mouse (Fig. 5B), suggesting that the distance from the E-box site to the transcription start site is conserved between species. The high conservation of the DNA sequence suggests that the interacting proteins on the DNA region are conserved. There are several E-boxes besides the MYC-responsive E-box near the promoter region. Thus, the characteristics proposed to be used for specific protein-DNA interaction, including high GC content (1), preferential spacing between transcription factor binding sites and transcription start sites (2), co-operativity of cis-regulatory molecules (3), and clustering of transcription factor binding sites near the target sites (4), are observed around this E-box site in the TAF4b promoter and may be involved in the mechanisms by which NCE-box 2 specifically functions as a MYC-target site.

The MYC responsive E-box in the TAF4b promoter is a noncanonical (CGCGTG) element. When the CGCGTG element was mutated to the canonical CACGTG, c-MYC still upregulated the promoter activity (Fig. 4A). While USF, another E-box-binding transcription factor, hardly stimulated the wild-type TAF4b promoter, it up-regulated the activity of the promoter with the CACGTG element (Fig. 4C). These results suggest that in addition to the context where the target E-box exists, a sequence within an E-box is involved in the mechanisms by which the E-box site is selected specifically by MYC.

When the CGCGTG element was mutated to the canonical CACGTG, c-MYC more strongly up-regulated the promoter activity (Fig. 4A). It was shown that c-MYC binds to CACGTG E-box element with higher affinity than other Eboxes (37). The decreased sensitivity of the non-canonical E-box with lower affinity to MYC may contribute to the proper gene activation by MYC. This idea is consistent with a recent study, which indicates that the affinity of binding sites is a primary determinant of the level of physiological stimulus necessary for substantial gene activation, contributing to finetuning the responses (38). TFE3, another E-box-binding transcription factor, activated the TAF4b promoter with neither CACGTG element nor CGCGTG element (Fig. 4B). Together, these results indicate that the sequences within E-box differently affect the activities of E-box-binding transcription factors and function as a factor to determine the spectrum of genes stimulated by the transcription factors.

TAF 4b is a Myc-target gene. In eukaryotes, protein-encoding genes are transcribed by RNA polymerase II (RNA Pol II). The molecular machinery that guides RNA Pol II to initiate transcription of a specific gene is composed of multiple classes of regulatory proteins (39). TFIID is a core component of the RNA Pol II regulatory machinery, which is conserved from Sacchoromyces cervisiae to humans and is a large multiprotein complex composed of the TATA box-binding protein (TBP) and several TBP-associated factors (TAFs). Although TFIID was initially thought to be ubiquitous in expression and function, identification of tissue-specific TAFs suggested that a specialized TFIID complex could play a direct role in regulating tissue-specific programs of gene expression. The first cell type-specific TFIID subunit identified was TAF4b 
(previously called $\mathrm{TAF}_{\mathrm{II}} 105$ ) (27), which is a homologous protein of a ubiquitously expressed factor, TAF4 (previously called $\left.\mathrm{TAF}_{\mathrm{II}} 130\right)$. The comparison between the three dimensional structure of the TFIID complex with TAF4b and that with original TAF4 suggest that distinct localizated structural changes in TFIID may cause a difference in their activity (40). Indeed, it was reported that TAF4b and TAF4 proteins share a common non-redundant function in regulating gene expression control $(41,42)$. These findings show that the expression control of TAF4b is a physiologically important issue. However, there have been no studies on the mechanisms of the TAF4b expression control.

It is well established that MYC is a central regulator of cell growth. There are studies describing functions and expression of TAF4b, which suggest the functional and expressional connection between TAF4b and MYC. In adult mouse testes, TAF4b was required for germ cell proliferation (43), and the expression of TAF4b and c-myc was detected in premeiotic spermatogonia (43-45). When primary B lymphocytes were activated by bacterial lipopolysaccharide (LPS), expression of TAF4b (46) and c-myc (47) was increased. TAF4b can induce a number of genes involved in the control of cell growth (41). It was also reported that TAF4b elevates the expression of VEGF (42), while vasculogenesis and angiogenesis were induced by MYC, which can indirectly induce the expression of VEGF (48). Therefore, TAF4b may be involved in the induction of VEGF expression by MYC (49). Together, the above studies suggest that the induction of TAF4b induced by MYC may contribute to cell growth control and tumorigenesis.

\section{Acknowledgements}

We thank Martin Eilers (Philipps-Universität Marburg) for providing pCMV-USF and pCMV-TFE3. We thank Kathryn E. Boyd and Peggy J. Farnham (University of Wisconsin Medical School) for sharing their protocols for chromatin immunoprecipitation analysis. This study was supported by Fukuoka Gann, Sankyo-Foundation and Grants-in-Aid from the Ministry of Education, Science and Culture of Japan.

\section{References}

1. Ioshikhes IP and Zhang MQ: Large-scale human promoter mapping using CpG islands. Nat Genet 26: 61-63, 2000.

2. Marino-Ramirez L, Spouge JL, Kanga GC and Landsman D: Statistical analysis of over-represented words in human promoter sequences. Nucleic Acids Res 32: 949-958, 2004.

3. Istrail S, De-Leon SB and Davidson EH: The regulatory genome and the computer. Dev Biol 310: 187-195, 2007.

4. Zhang C, Xuan Z, Otto S, Hover JR, McCorkle SR, Mandel G and Zhang MQ: A clustering property of highly-degenerate transcription factor binding sites in the mammalian genome. Nucl Acids Res 34: 2238-2246, 2006.

5. DePinho RA, Schreiber-Agus N and Alt FW: $m y c$ family oncogenes in the development of normal and neoplastic cells. Adv Cancer Res 57: 1-46, 1991

6. Grandori C, Cowley SM, James LP and Eisenman RN: The $\mathrm{Myc} / \mathrm{Max} / \mathrm{Mad}$ network and the transcriptional control of cell behavior. Annu Rev Cell Dev Biol 16: 653-699, 2000.

7. Henriksson M and Luscher B: Proteins of the Myc network: essential regulators of cell growth and differentiation. Adv Cancer Res 68: 109-182, 1996

8. Marcu KB, Bossone SA and Patel AJ: myc function and regulation. Annu Rev Biochem 61: 809-860, 1992.
9. Morgenbesser SD and DePinho RA: Use of transgenic mice to study $m y c$ family gene function in normal mammalian development and in cancer. Semin Cancer Biol 5: 21-36, 1994.

10. Davis AC, Wims M, Spotts GD, Hann SR and Bradley A: A null c-myc mutation causes lethality before 10.5 days of gestation in homozygotes and reduced fertility in heterozygous female mice. Genes Dev 7: 671-682, 1993.

11. Sawai S, Shimono A, Wakamatsu Y, Palmes C, Hanaoka K and Kondoh H: Defects of embryonic organogenesis resulting from targeted disruption of the N-myc gene in the mouse. Development 117: 1445-1455, 1993.

12. Stanton BR, Perkins AS, Tessarollo L, Sassoon DA and Parada LF: Loss of N-myc function results in embryonic lethality and failure of the epithelial component of the embryo to develop. Genes Dev 6: 2235-2247, 1992.

13. Trumpp A, Refaell Y, Oskarsson T, Gasser S, Murphy M, Martin GR and Bishop JM: c-Myc regulates mammalian body size by controlling cell number but not cell size. Nature 414: 768-773, 2001 .

14. Luscher B: Function and regulation of the transcription factors of the Myc/Max/Mad network. Gene 277: 1-14, 2001.

15. Dang CV: c-Myc target genes involved in cell growth, apoptosis, and metabolism. Mol Cell Biol 19: 1-11, 1999.

16. Levens DL: Reconstructing MYC. Genes Dev 17: 1071-1077, 2003

17. Bello-Fernandez C, Packham G and Cleveland JL: The ornithine decarboxylase gene is a transcriptional target of c-Myc. Proc Natl Acad Sci USA 90: 7804-7808, 1993.

18. Tsuneoka M, Nakano F, Ohgusu H and Mekada E: c-myc activates RCC1 gene expression through E-box elements. Oncogene 14: 2301-2311, 1997.

19. Greasley PJ, Bonnard C and Amati B: Myc induces the nucleolin and BN51 genes: possible implications in ribosome biogenesis Nucl Acids Res 28: 446-453, 2000.

20. Bouchard C, Dittrich O, Kiermaier A, Dohmann K, Menkel A, Eilers $M$ and Luscher B: Regulation of cyclin D2 gene expression by the Myc/Max/Mad network: Myc-dependent TRRAP recruitment and histone acetylation at the cyclin D2 promoter. Genes Dev 15: 2042-2047, 2001.

21. Teye K, Tsuneoka M, Arima N, Koda Y, Nakamura Y, Ueta Y, Shirouzu K and Kimura H: Increased expression of a Myc target gene Mina53 in human colon cancer. Am J Pathol 164: 205-216, 2004.

22. Tsuneoka M, Fujita H, Arima N, Teye K, Okamura T, Inutsuka H, Koda Y, Shirouzu $\mathrm{K}$ and Kimura $\mathrm{H}$ : Mina53 as a potential prognostic factor for esophageal squamous cell carcinoma. Clin Cancer Res 10: 7347-7356, 2004.

23. Tsuneoka M, Koda Y, Soejima M, Teye K and Kimura H: A novel Myc target gene, mina53, that is involved in cell proliferation. J Biol Chem 277: 35450-35459, 2002.

24. Tsuneoka M, Teye K, Arima N, Soejima M, Otera H, Ohashi K, Koga Y, Fujita H, Shirouzu K, Kimura H and Koda Y: A novel Myc-target gene, mimitin, that is involved in cell proliferation of esophageal squamous cell carcinoma. J Biol Chem 280: 19977-19985, 2005

25. Gregor PD, Sawadogo M and Roeder RG: The adenovirus major late transcription factor USF is a member of the helix-loophelix group of regulatory proteins and binds to DNA as a dimer. Genes Dev 4: 1730-1740, 1990.

26. Beckmann H, Su LK and Kadesch T: TFE3: a helix-loop-helix protein that activates transcription through the immunoglobulin enhancer muE3 motif. Genes Dev 4: 167-179, 1990.

27. Dikstein R, Zhou $S$ and Tijian R: Human $\mathrm{TAF}_{\mathrm{II}} 105$ is a cell type-specific TFIID subunit related to $\mathrm{hTAF}_{\mathrm{II}} 130$. Cell 87: 137-146, 1996.

28. Desbarats L, Gaubatz S and Eilers M: Discrimination between different E-box-binding proteins at an endogenous target gene of c-myc. Genes Dev 10: 447-460, 1996.

29. Eilers M, Picard D, Yamamoto KR and Bishop JM: Chimaeras of Myc oncoprotein and steroid receptors cause hormonedependent transformation of cells. Nature 340: 66-68, 1989

30. Hickstein DD, Back AL and Collins SJ: Regulation of expression of the CD11b and CD18 subunits of the neutrophil adherence receptor during human myeloid differentiation. J Biol Chem 264: 21812-21817, 1989.

31. Hozumi M: Fundamentals of chemotherapy of myeloid leukemia by induction of leukemia cell differentiation. Adv Cancer Res 38: 121-169, 1983.

32. Vass JK, Neil R, Jamieson T and Birnie GD: Regulation of the relative abundances of c-myc mRNA in human promyelocytic HL60 cells. Differentition 45: 49-54, 1990. 
33. Coller HA, Grandori C, Tamayo P, Colbert T, Lander ES, Eisenmn RN and Golub TR: Expression analysis with oligonucleotide microarrays reveals that MYC regulates genes involoved in growth, cell cycle, signaling, and adhesion. Proc Natl Acad Sci USA 97: 3260-3265, 2000

34. Grandori C, Mac J, Siebelt F, Ayer DE and Eisenman RN: Myc-Max heterodimers activate a DEAD box gene and interact with multiple E box-related sites in vivo. EMBO J 15: 4344-4357, 1996.

35. Boyd KE and Farnham PJ: Myc versus USF: discrimination at the $c a d$ gene is determined by core promoter elements. Mol Cell Biol 17: 2529-2537, 1997

36. Boyd KE, Wells J, Gutman J, Bartley SM and Farnham PJ: c-Myc target gene specificity is determined by a post-DNAbinding mechanism. Proc Natl Acad Sci USA 95: 13887-13892, 1998.

37. Blackwell TK, Huang J, Ma A, Kretzner L, Alt FW, Eisenman RN and Weintraub $\mathrm{H}$ : Binding of myc proteins to canonical and noncanonical DNA sequences. Mol Cell Biol 13: 5216-5224, 1993.

38. Lam FH, Steger DJ and O'Shea EK: Chromatin decouples promoter threshold from dynamic range. Nature 453: 246-250, 2008.

39. Lemon B and Tijian R: Orchestrated response: a symphony of transcription factors for gene control. Genes Dev 14: 2551-2569, 2000 .

40. Liu WL, Coleman RA, Grob P, King DS, Florens L, Washburn MP, Geles KG, Yang JL, Ramey V, Nogales E and Tjian R: Structural changes in TAF4b-TFIID correlate with promoter selectivity. Mol Cell 29: 81-91, 2008.

41. Mengus G, Fadloun A, Kobi D, Thibault C, Perletti L, Michel I and Davidson I: TAF4 inactivation in embryonic fibroblasts activates TGFbeta signalling and autocrine growth. EMBO J 24: 2753-2767, 2005
42. Geles KG, Freiman RN, Liu W-L, Zheng S, Voronina E and Tjian R: Cell-type-selective induction of c-jun by TAF4b directs ovarian-specific transcription networks. Proc Natl Acad Sci USA 103: 2594-2599, 2006.

43. Falender AE, Freiman RN, Geles KG, Lo KC, Hwang K, Lamb DJ, Morris PL, Tjian R and Richards JS: Maintenance of spermatogenesis requires TAF4b, a gonad-specific subunit of TFIID. Genes Dev 19: 794-803, 2005.

44. Wolfes H, Kogawa K, Millette CF and Cooper GM: Specific expression of nuclear proto-oncogenes before entry into meiotic prophase of spermatogenesis. Science 245: 740-743, 1989.

45. Koji T, Izumi S, Tanno M, Moriuchi T and Nakane PK: Localization in situ of c-myc mRNA and c-myc protein in adult mouse testis. Histochem J 20: 551-557, 1998.

46. Freiman RN, Albright SR, Chu LE, Zheng S, Liang H-E, Sha WC and Tijian R: Redundunt role of tissue-selective $\mathrm{TAF}_{\mathrm{II}} 105$ in $\mathrm{B}$ lymphocytes. Mol Cell Biol 22: 6564-6572, 2002.

47. Kelly K, Cochran BH, Stiles CD and Leder P: Cell-specific regulation of the c-myc gene by lymphocyte mitogens and platelet-derived growth factor. Cell 35: 603-610, 1983.

48. Baudino TA, McKay C, Pendeville-Samain H, Nilsson JA, Maclean KH, White EL, Davis AC, Ihle JN and Cleveland JL: $\mathrm{c}-\mathrm{Myc}$ is essential for vasculogenesis and angiogenesis during development and tumor progression. Genes Dev 16: 2530-2543, 2002.

49. Pelengaris S, Khan M and Evan GI: Suppression of Myc-induced apoptosis in beta cells exposes multiple oncogenic properties of Myc and triggers carcinogenic progression. Cell 109: 21-34, 2002. 